\title{
The Landscape of Medical Literature in the Era of COVID-19: Original Research Versus Opinion Pieces
}

J Gen Intern Med 35(9):2813-5

DOI: $10.1007 / \mathrm{s} 11606-020-06021-8$

(c) Society of General Internal Medicine 2020

\section{INTRODUCTION}

There has been rapidly growing scientific literature related to the coronavirus disease 2019 (COVID-19), in attempts to convey any potential hypotheses or confirmed facts about the disease. Notably, there has been a plethora of viewpoints and other opinion pieces to help guide ongoing research efforts. However, this trend carries a risk of redundant publications. ${ }^{1,2}$ Our objective was to evaluate the nature of the published scientific literature (original investigations and opinion pieces) related to COVID-19 and to quantify their relative proportions to determine whether the trend of publication of opinion pieces has changed over time.

\section{METHODS}

For this cross-sectional analysis, we searched the Medline database from February 1, 2020, to April 20, 2020, using the keywords "COVID-19" and "corona." Articles were categorized to "opinion" articles (i.e., narrative reviews, viewpoints, perspectives, commentaries, news pieces, letters to the Editor, educational material, and consensus statements/guidelines), and original investigations (i.e., randomized control trials [RCTs], observational studies [retrospective, cross-sectional or prospective], meta-analyses, translational or animal studies, and epidemiologic modeling studies). Consensus statements and guidelines were considered as "opinion" articles since the recommendations from these documents are mainly based on expert opinion. Case reports and case series of $\leq 10$ patients as well as articles describing a clinical trial design or a prospective study protocol were excluded. Editorials addressing articles published in the same journal issue were excluded since those are integral part of the journal structure. In addition, we excluded systematic reviews given the lack of consensus on whether they should be considered as original investigations. ${ }^{3}$ Non-English articles

Akram Y. Elgendy and Amr F. Barakat contributed equally to this work.

Received June 2, 2020

Accepted June 30, 2020

Published online July 8, 2020 where the exact type could not be determined by reviewing the title and abstract were also excluded. Importantly, to ensure that articles are classified into the correct categories, we manually reviewed the content of each article rather than depending on the provided journal classifications. Analysis of variance (ANOVA) was used to assess the trend in the relative proportion of "opinion" articles over the study period. Descriptive statistical analyses were performed using SPSS statistical package (SPSS version 25.0, IBM Inc., Armonk, NY).

\section{RESULTS}

The search identified 5479 articles, among which 276 were non-COVID-related, 63 were non-English articles that could not be classified, 8 were errata, and 3 articles were retracted. In addition, 403 were case reports or case series of $\leq 10$ patients, 89 were systematic reviews, 20 were trial design or study protocol, and 124 were editorials related to an article within the same journal. The final analysis included 4493 articles. Among 1030 (22.9\%) original investigations, the majority were retrospective or cross-sectional observational studies (57.9\%). Most of the observational studies $(93.4 \%)$ were related to diagnosis of the disease. Translational or animal studies comprised $16.8 \%$ of original investigations, epidemiological modeling analyses $14.8 \%$, prospective observational studies $5.7 \%$, meta-analyses $4.6 \%$, and RCTs $0.3 \%$. The number of "opinion" articles was 3463 $(77.1 \%)$ (Table 1). The ratio of "opinion" articles to original investigations was $3.4: 1$. There was no significant change in the trend of the relative proportion of "opinion" articles to original investigations throughout the study period when divided into quintiles from oldest versus latest, $R^{2}$ for quadratic trend model was 0.74 and corresponding $p$ value for regression was 0.3 (Figure 1).

\section{DISCUSSION}

In this cross-sectional analysis of published scientific articles related to COVID-19 in the Medline database over an 80-day period, we found that the number of "opinion" articles was $>3$ times the number of original investigations published in this time frame. Interestingly, there was no significant change in the temporal trend of this ratio, which illustrates that the 
Table 1 Types of Articles Included in the Analysis

\begin{tabular}{ll}
\hline \hline & $\begin{array}{l}\text { Number } \\
(\%)\end{array}$ \\
\cline { 2 - 2 } & $\mathbf{4 4 9 3}$ \\
\hline Original investigations & $1030(22.8)$ \\
Randomized controlled trial & $3(0.07)$ \\
Observational study & $655(14.5)$ \\
Prospective, number (\% of observational studies) & $59(9.0)$ \\
Retrospective/cross-sectional, number (\% of & $596(91.0)$ \\
observational studies) & \\
Related to disease diagnosis, number (\% of & $612(93.4)$ \\
observational studies) & $43(6.6)$ \\
$\begin{array}{l}\text { Evaluating a therapeutic intervention, number (\% of } \\
\text { observational studies) }\end{array}$ & $473(3.9)$ \\
Translational or animal study & $152(3.4)$ \\
Epidemiologic modeling analysis & $47(1.0)$ \\
Meta-analysis & $3463(77.1)$ \\
Opinion articles & $365(8.1)$ \\
Narrative review & $140(3.1)$ \\
Society guideline or consensus statement & $81(1.8)$ \\
Non-society multicenter guideline or consensus state- & \\
ment & $2614(58.2)$ \\
Viewpoint, perspective, commentary or news piece & $256(5.7)$ \\
Letter to the Editor/Response to a letter to the Editor & $7(0.2)$ \\
Patient education material & \\
\hline
\end{tabular}

number of "opinion" articles remains high. Since our knowledge and understanding of this disease are premature, there is an understandable need for valuable insights to guide research efforts. However, this analysis suggests that the number of these "opinion" articles continues to be high, and there might be some concerns for redundant publications. For example, there has been $>10$ viewpoints related to renin-angiotensinaldosterone inhibitors and COVID-19 published in several journals even before any original data about the topic became available. $^{4,5}$

This study is limited by search of one online database at a single time point. There are many ongoing research efforts and this trend is expected to change with time. Based on the findings from this analysis, medical journals might want to re-evaluate the usefulness of COVID-19-related opinion pieces being considered for publication.

Akram Y. Elgendy, $M D^{1}$

Amr F. Barakat, $\mathrm{MD}, \mathrm{MSc}^{2}$

Joseph Ibrahim, $\mathrm{MD}^{3}$

Laith Alkukhun, $\mathrm{MD}^{2}$

Mamas A. Mamas, BM, BCh, DPhil ${ }^{4}$

Islam Y. Elgendy, MD, FACC, FAHA, FSCAI, FESC, FACP ${ }^{5}$

${ }^{1}$ Division of Cardiovascular Medicine, University of California San Fransisco,

San Fransisco, CA, USA

${ }^{2}$ Heart and Vascular Institute, University of Pittsburgh Medical Center,

Pittsburgh, PA, USA

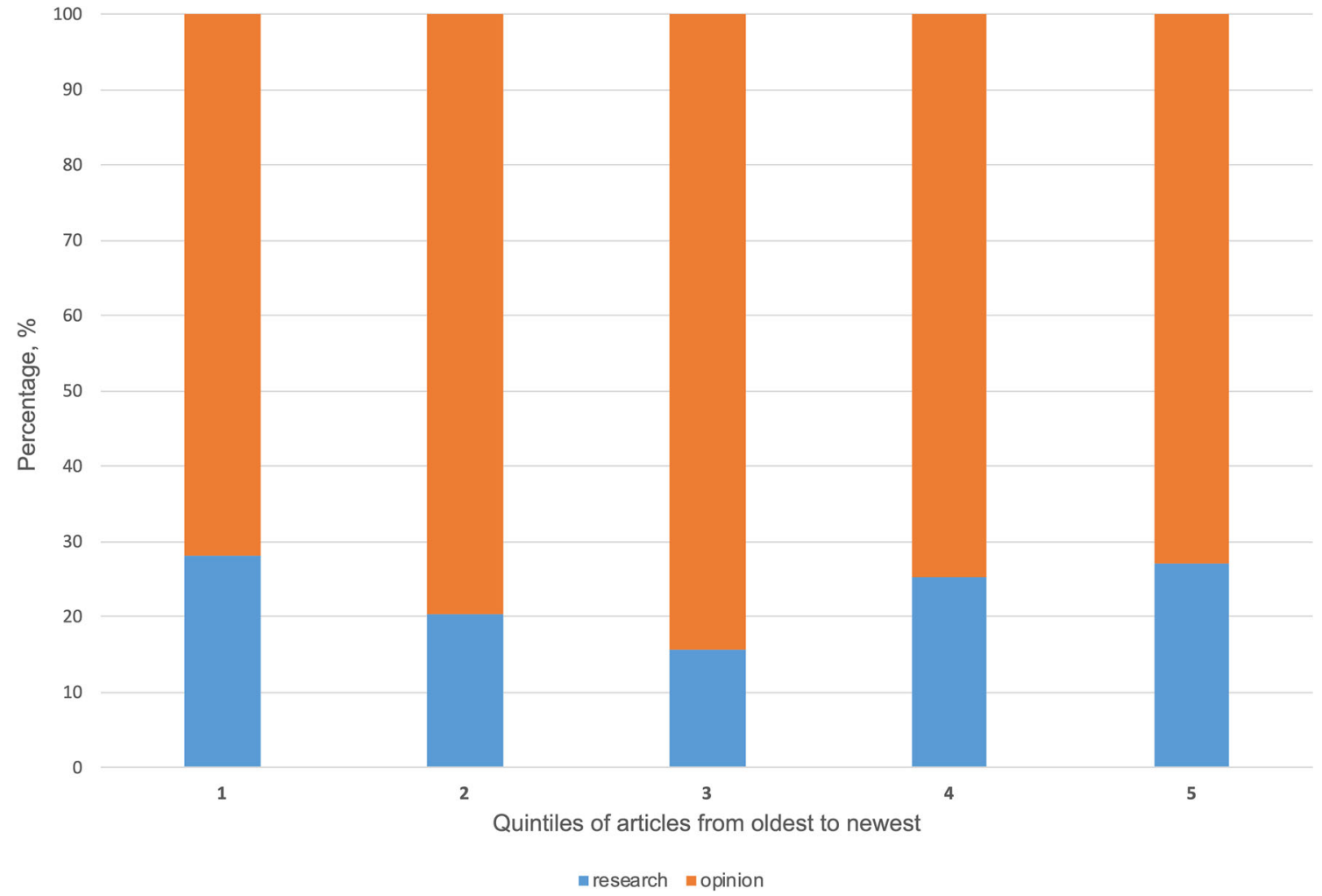

Figure 1 The relative proportion of "opinion" articles (blue) to original investigations (orange) throughout the study period. The total number of articles included in the analysis was divided chronologically into quintiles from oldest (1) to newest (5). 
${ }^{3}$ Department of Internal Medicine, University of Pittsburgh Medical Center,

Pittsburgh, PA, USA

${ }^{4}$ Keele Cardiovascular Research Group, Keele University,

Stoke-on-Trent , UK

${ }^{5}$ Division of Cardiology, Weill Cornell Medicine-Qatar, Doha, Qatar

Corresponding Author: Islam Y. Elgendy, MD, FACC, FAHA, FSCAI, FESC, FACP; Division of Cardiology, Weill Cornell Medicine-Qatar, Doha, Qatar (e-mail: iyelgendy@gmail.com).

\section{REFERENCES}

1. Johnson C. Repetitive, duplicate, and redundant publications: a review for authors and readers. J Manip Physiol Ther 2006;29:505-509.
2. Yank V, Barnes D. Consensus and contention regarding redundant publications in clinical research: Cross-sectional survey of editors and authors. J Med Ethics 2003;29:109-114.

3. Krnic Martinic M, Meerpohl JJ, von Elm E, Herrle F, Marusic A, Puljak L. Attitudes of editors of core clinical journals about whether systematic reviews are original research: a mixed-methods study. BMJ Open 2019;9:e029704.

4. Vaduganathan M, Vardeny $\mathbf{O}$, Michel T, McMurray JJV, Pfeffer MA, Solomon SD. Renin-angiotensin-aldosterone system inhibitors in patients with Covid-19. N Engl J Med 2020;382:1653-1659.

5. Patel AB, Verma A. COVID-19 and Angiotensin-converting enzyme inhibitors and angiotensin receptor blockers: What is the evidence? JAMA. 2020. doi:https://doi.org/10.1001/jama.2020.4812

Publisher's Note Springer Nature remains neutral with regard to jurisdictional claims in published maps and institutional affiliations. 